\title{
Efeitos do abandono em áreas agrícolas marginais: reflexos na dinâmica da vegetação $^{1}$
}

\author{
Albano Figueiredo \\ Instituto de Estudos Geográficos. Faculdade de Letras. Universidade de Coimbra. \\ geofigsfl.uc.pt
}

\section{Carlos Aguiar}

Escola Superior Agránia. Instituto Politécnico de Bragança.

cfaguiaryipb.pt

\section{Resumo}

Apresenta-se uma análise floristica e estrutural das formaçōes arbustivas dominantes instaladas após processos de abandono agricola em substratos graniticos e xistentos, na transição entre a região Eurossiberiana e a Região Mediterrânea. Direcciona-se esta análise a formaçōes arbustivas microfanerofiticas dominadas pelo género Cytisus, dada a elevada frequência com que estas comunidades ocorrem em parcelas agrícolas abandonadas, como se pode verificar pela importância dos giestais em sectores da paisagem dominados pelo abandono agrícola. Procede-se ainda a um enquadramento destas comunidades no processo de sucessão vegetal, à análise do seu significado em relação às séries de vegetação presentes e identificação das condições ecológicas responsáveis pela instalação destas comunidades interpretadas como basais, nomeadamente as dominadas por Cytisus multiflorus (L'Hér.) Sweet e por Cytisus striatus (Hill) Rothm..

Palavras-chave: Abandono agrícola. Sucessão vegetal. Giestais. Cytisus sp.

\section{Résumé}

Conséquences de l'abandon aux paysages marginaux: effets sur la dynamique de la végétation

L'abandon de l'agriculture aux paysages d'utilisation marginale apporte des changements très importants sur la végétation, parce qu'il conduit à la dominance des formations arbustives. Cet article présent une analyse floristique et physionomique de ces communautés aux secteurs affectés par ce phénomène dans la transition entre les Régions Méditerranéenne et Eurosibérienne de l'extrême Nord du Portugal.

En se basant sur les substrats acides, cette étude a analysé les communautés les plus fréquentes qui, dans ce territoire, sont dominées par des espèces du genre Cytisus. Associées à une colonisation très rapide, les communautés à Cytisus striatus (Hill) Rothm. et à Cytisus multiflorus (L'Hér.) Sweet sont, du point de vu floristique, très pauvres, et leur implantation est contrôlée par les conditions édaphiques.

Mots-clés: Abandon agricole. Succession. Communautés arbustives. Cytisus sp.

\section{Abstract}

Consequences of abandonment in marginal landscapes: effects on vegetation dynamics

The consequences of abandonment on marginal landscapes lead to important changes on vegetation, allowing the dominance of shrub formations. This article presents a floristic and physiognomic analysis of dominant shrub formations related to abandonment of agriculture activities. On siliceous substrate, at the border between Eurosiberian and Mediterranean floristic regions, such analysis is directed to shrub formations dominated by Cytisus genus, taking its frequency and association with abandonment. Besides its interpretation on vegetation dynamics, is also demonstrated its connection with present vegetation series and determinant ecological conditions for the presence of communities dominated by Cytisus multiflorus (L'Hér.) Sweet or Cytisus striatus (Hill) Rothm..

Key-Words: Abandonment. Succession. Broom communities. Cytisus sp.

\footnotetext{
'Estudo realizado no âmbito do Projecto POCTI/GEO/49371/2002, financiado pela Fundaçảo para a Ciência e a Tecnologia (FCT) e Fundos FEDER.
} 
Introdução

O abandono agrícola, acompanhado por um vasto conjunto de modificações ao nível do uso do solo, apresenta importantes reflexos na paisagem rural. No âmbito da Península Ibérica os processos de abandono e respectivas causas e consequências foram alvo de diversos estudos (PÉrEz-ChÁCON e VABRE, 1988; LLORENTE e LUENGO, 1986), principalmente em áreas montanhosas, onde o abandono assume grande magnitude. A menor disponibilidade destes estudos para o território continental português pode dever-se a um desfasamento temporal em termos de abandono definitivo e seus reflexos, uma vez que este abandono foi intercalado por processos de reconversão em termos de usos do solo, no sentido de maior extensividade, o que protelou as consequências de um abandono definitivo. Os estudos feitos para o território nacional versam essencialmente sobre uma vertente sócio-demográfica, revelando especial preocupação com as causas deste fenómeno (CORREIA, 2006). Relegam-se para segundo plano a análise dos reflexos nos sistemas naturais, nomeadamente ao nível da dinâmica sucessional da vegetação e as suas consequências para o espaço florestal, bem como a definição de medidas de gestão adequadas a esta realidade. Quanto às causas, especialmente no seu sector interior Norte e Centro, os processos de abandono estão muito associados a dinâmicas sócio-demográficas específicas, nomeadamente à ocorrência de movimentos migratórios internos (êxodo rural) e externos (emigração), os quais foram responsáveis pelo esvaziamento populacional destas áreas e o consequente definhamento económico. A par deste esvaziamento populacional verificou-se um reforço do envelhecimento e a carência de população activa, com reflexos no abandono das actividades primárias. 0 abandono agrícola favoreceu um aumento importante de parcelas sem uma utilização definida, muitas vezes associadas a processos de pousio mais prolongados. As parcelas que deixaram de estar associadas a uma utilização periódica permitiram a reactivação dos processos de sucessão e a instalação de comunidades arbustivas, frequentemente perturbadas pelo fogo em áreas onde o processo de abandono assumiu uma extensão espacial mais importante. Esta sucessão secundária está, em termos de cortejo florístico e velocidade dos processos sucessionais, determinada pelas condições ecológicas das parcelas, factor condicionante do tipo, duração e intensidade da perturbação anteriormente verificada.

0 decurso da sucessão vegetal nas parcelas agricolas abandonadas está associado à instalação de comunidades arbustivas nos estádios intermédios, frequentemente dominadas por leguminosas fabáceas da tribo Cytiseae. 0 seu carácter heliófilo e boas taxas de germinação em função de temperaturas mais elevadas ao nivel do solo (TÁRREGA et al., 1992; MARCos et al., 2004) funcionam como estímulos adequados ao seu domínio em processos de sucessão secundária, favorecendo extensa representatividade na paisagem do interior norte e centro do país (COSTA et al., 2003).

\section{Metodologia e área em estudo}

A área em estudo está dominada, topográfica e geomorfologicamente, pela depressão de Chaves, a qual representa uma importante interrupção orográfica entre os sectores culminantes da Padrela a Este e as Alturas do Barroso a Oeste. Esta unidade geomorfológica faz parte de um conjunto de depressões que estão geneticamente associadas ao acidente tardivarisco (NNE-SSW) Verin-Penacova.

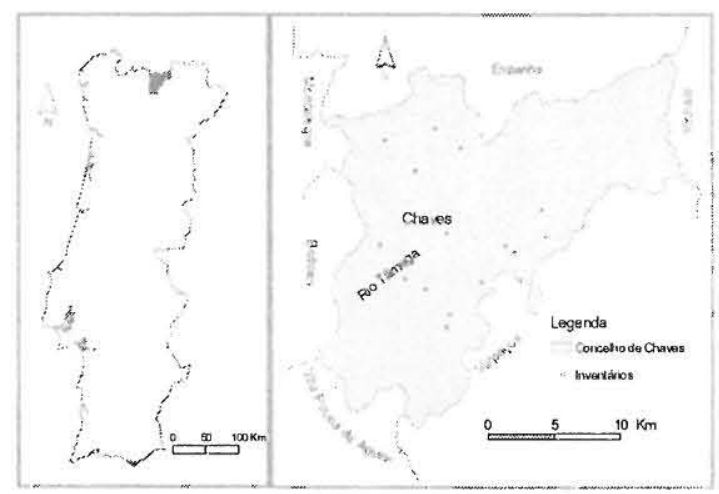

Figura1

Localização da área em estudo e locais de inventariação

Incluída na região Mediterrânea, a área em estudo confina a ocidente com a região Eurossiberiana, situação que se identifica na presença de alguns taxa associados à influência atlântica (Agrostis curtisii, Daboecia cantabrica, Quercus robur). Definida no Sector Orensano-Sanabriense (COSTA et al., 1998), desenvolve-se entre as condições mesomediterrâneas sub-húmidas, do fundo da depressão e sectores de vertente desenvolvidos até aos $700 \mathrm{~m}$, e as situações hiper-húmidas dos sectores supramediterrâneos de maior altitude do flanco ocidental da depressão.

Tendo em conta a sua particular configuração geomorfológica, o uso do solo está muito determinado pelas condições topográficas e de substrato, nomeadamente pelos declives acentuados de vertentes predominantemente graníticas, definidos como elementos essenciais no controlo das condições ecológicas. Deste modo, é possível definir diferentes unidades quanto à 
importância do uso agricola, intensidade de perturbação e magnitude do abandono.

Classificou-se a área em estudo quanto à importância da actividade agrícola, intensidade de perturbação, magnitude do abandono, e o tipo de comunidades arbustivas associadas a situações de abandono, de forma a concentrar a análise nas comunidades mais frequentes. Neste sentido chegou-se à seguinte classificação:

a) Planície aluvial actual do Tâmega e depósitos aluvio-coluvionares do fundo da depressão: declive muito fraco ou ausente, onde a ocupação agrícola é máxima, o que implica uma situação de máxima perturbação, sendo as situações de abandono muito restritas espacialmente e associadas a condições ecológicas muito especificas. Identificam-se apenas situaçōes de abandono em solos com encharcamento prolongado, permitindo a instalação de comunidades dominadas por Molinia caerulea, cuja degradação está assegurada pela instalação de um salgueiral de Salix atrocinerea e S. neotricha. Em sectores com encharcamento mais limitado no tempo, estas formações herbáceas são degradadas pela instalação de um tojal, que integra Ulex minor e Genista anglica, após abandono de parcelas anteriormente perturbadas por herbivoria e produção de feno, e que antecede a instalação de comunidades altifruticetas associadas à Rhamno-Prunetea (RODRIGUES, 2005).

b) Flancos da depressão de Chaves: os declives acentuados condicionam fortemente a prática agricola, sendo um tipo de perturbação com fraca representatividade, frequentemente associada a socalcos. O dominio de formações arbustivas está fortemente associado à perturbação pelo fogo e resiliência das mesmas, uma vez que os acentuados declives e solos delgados não favorecem uma sucessāo rápida. Verifica-se o domínio de giestais brancos (Cytisus multiflorus), que por vezes cedem perante estevais (Cistus ladanifer) em solos decapitados por erosão em sectores termo-xerófilos.

c) Sectores correspondentes à superfície fundamental da meseta, níveis embutidos e outras áreas com declives fracos a médios (Superfície de Sanjurge, Nivel de Águas Frias ${ }^{2}$ ): neste sector o abandono agricola assume grande magnitude, em paralelo com situações de pousio prolongado. As formações arbustivas associadas ao abandono agricola assumem grande representatividade, especialmente as dominadas por espécies do género Cytisus.

2. Para mais informação sobre as condiçōes destas unidades

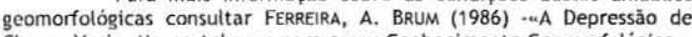
Chaves-Verin. Novas Achegas para o seu Conhecimento Geomorfológicon. In BRITO, Raquel Soeiro de (coord.) - Estudos de Homenagem a Mariano Feio. Instituto Nacional de Investigaçăo Científica, pp. 199-222.
A partir da classificação anterior centrou-se a análise nos sectores onde o abandono assume maior representatividade, mais especificamente nos sectores identificados em C, mediante a selecção de parcelas onde o decurso dos processos sucessionais permitiu a instalação de formaçōes arbustivas. A realização de 23 inventários fitossociológicos, segundo a metodologia proposta por Gèhu e Rivas-Marinez (1981), incidiu sobre as comunidades mais frequentes, pelo que se apresenta informação apenas para as comunidades dominadas por Cytisus striatus e $C$. multiflorus.

Em termos de sintaxonomia segue-se a proposta patente em RIVAS-MARTINEZ et al. (1999), RIVAS-MARTINEZ et al. (2001) e Rivas-MARTinez et al. (2002); a identificação, taxonomia e nomenclatura dos taxa baseou-se nas floras de Franco e ROCHA AFOnso (1998) e CASTROVIEJo et al. (1986-2000); a nomenclatura biogeográfica segue a proposta de CosTA et al. (1998).

Tendo em conta a representatividade destas formações microfanerofíticas, dominantes na paisagem agricola resultante de abandono, recolheu-se informação necessária a uma caracterização ecológica que permita distinguir os factores que promovem a instalação de um giestal amarelo, onde domina Cytisus striatus, ou de um giestal branco, dominado por Cytisus multiflorus. Para esta caracterização recolheram. se amostras de solo ${ }^{3}$ (16 amostras) para análise de textura (análise granulométrica ${ }^{4}$ ), $\mathrm{pH}$, teor de matéria orgânica, K e P e informação sobre a litologia, declive e altitude, de forma a identificar factores ecológicos determinantes.

\section{Discussão de resultados}

$\mathrm{Na}$ paisagem agricola resultante de abandono verifica-se um domínio claro de formações arbustivas altas dominadas por Cytisus striatus, sendo menos frequente a definição de comunidades dominadas por Cytisus multiflorus. Ambas correspondem a comunidades basais de elevada pobreza florística, situaçōes de insaturação fitocenótica ${ }^{5}$, interpretadas como desvios na sucessão em relação às comunidades arbustivas subseriais, no âmbito do domínio climácico do Holcus mollis-Quercetum pyrenaicae sigmetum. São entendidas como comunidades primocolonizadoras de solos abandonados pela agricultura ${ }^{6}$, evidenciando empobre-

3. A recolha das amostras de solo è feita nos $20 \mathrm{~cm}$ superficiais, após remoçāo dos horizontes orgânicos (ADO e AO).

4. $\mathrm{Na}$ análise granulométrica, e para partículas inferiores a $63 \mu$, foi utilizado granulómetro laser.

5 . Comunidades unicamente constituídas por espécies caracteristicas de unidades superiores à associação e companheiras (AGUIAR, 2001). correspondendo a situaçōes em que a utilizaçāo agricola, implicando 
cimento em relação a sintaxa subseriais, em função do controlo que a perturbação agrícola exerceu sobre os processos de dinâmica sucessional.

Relacionadas com dinâmicas sucessionais progressivas, estas comunidades nano-microfanerofíticas de carácter heliófilo instalam-se em curtos períodos de tempo (5-10 anos), estabelecendo um controlo imediato do espaço.

O papel das condições edáficas na definição das comunidades arbustivas

As condições do solo são de capital importância no controlo do cortejo florístico das comunidades arbustivas instaladas após abandono agrícola, desempenhando a litologia o papel determinante no seu condicionamento.

Os giestais altos e densos dominados por Cytisus striatus são os mais comuns, sendo as comunidades dominadas por Cytisus multiflorus pouco frequentes em dinâmicas progressivas a decorrer em parcelas agricolas abandonadas, apesar de frequentes na paisagem. Esta situação está relacionada com o facto de as parcelas ocupadas pela agricultura apresentarem condiçōes edáficas mais favoráveis à instalação de comunidades arbustivas dominadas por Cytisus striatus, devido à presença de solos mais profundos e com melhores potencialidades. O predomínio de Cytisus striatus está ainda associado a aspectos como a estrutura física e longevidade desta espécie, crescimento rápido, elevada produção de sementes aliados à possibilidade de constituir povoamentos densos, monoespecíficos, estratégias de taxa com comportamento invasor, o qual está identificado em territórios onde não é nativa, como na Califórnia (CIPC, 2006).

As comunidades dominadas por Cytisus multiflorus, menos associadas ao abandono agrícola directo, remetem-se, na paisagem, para biótopos caracterizados por solos graníticos pobres, muito delgados, onde a escassez de recursos hídricos é reforçada pela textura arenosa, declives acentuados e frequência de afloramentos rochosos. Quando associado ao abandono agrícola, o giestal branco encontra-se exclusivamente representado em substratos graníticos, correspondendo a parcelas marginais anteriormente utilizadas em sistema de pousio prolongado, normalmente ligadas à cultura do centeio, dada a pobreza dos solos. $\mathrm{Da}$ análise das condições edáficas destaca-se a textura predominantemente arenosa, em que a fracção fina
(< 62 $\mu$ ) representa normalmente menos de $15 \%$ na composição ganulométrica.

Em substratos graníticos, em cambissolos profundos de textura franco-arenosa, onde a ausência de declive é acompanhada por um espessamento do horizonte arável (antropogénico), os giestais brancos são substituídos por comunidades dominadas por Cytisus striatus, como se verifica nos sectores supramediterrâneos. São comunidades normalmente mais abertas e de maior diversidade florística, em relação às desenvolvidas em xistos, cujo porte mais alto $\mathrm{e}$ maior densidade favorecem maior empobrecimento florístico. A presença destes giestais em substratos graníticos supramediterrâneos pode estar associada, na área em estudo, a um reforço da influência atlântica, traduzida em totais pluviométricos mais elevados. Esta ideia reforça-se na comparação com o que se verifica nos sectores supramediterrâneos altibeirenses. Aqui, os giestais dominados por Cytisus striatus circunscrevem-se a substratos xistentos, deixando os substratos graniticos sob o domínio de um giestal branco, cujo cortejo florístico manifesta um reajuste no sentido do Thymo mastichinae-Cytisetum multiflori (Genistion floridae), de carácter mais continental, apenas presente na área em estudo em biótopos xerófilos sem perturbação agrícola directa.

Enquanto que os giestais domimados por Cytisus striatus ocorrem em solos siliciosos mais profundos e bem drenados (cambissolos húmicos e dístricos profundos), as comunidades dominadas por Cytisus multiflorus ocupam solos de menor espessura e textura arenosa (cambissolos dístricos pouco profundos). Pontualmente, mas com pouco significado espacial, é possivel encontrar comunidades dominadas por Adenocarpus complicatus s.l. (codeçais), em solos de génese coluvial e aluvio-coluvial.

Da análise efectuada para as restantes variáveis edáficas em análise não se identificou qualquer correlação directa significativa com o tipo de giestal.

\section{Enquadramento sintaxonómico}

CYTISETEA SCOPARIO-STRIATI Rivas-Martinez 1974

** Cytisetalia scopario-striati Rivas-Martinez 1974

* Ulici europaei-Cytision striati Rivas-Martinez, Báscones, Díaz, Fez-González e Loidi 1991

Comunidade basal de Cytisus striatus

Lavandulo sampaioanae-Cytisetum multiflori

Br.-Bl., P. Silva e Rozeira 1964 
As comunidades inventariadas são interpretadas no âmbito da aliança Ulici europaei - Cytision striati, que inclui formaçōes arbustivas de carácter silicícola, termo-mesotemperadas e mesomediterrâneas de ombroclimas sub-húmidos a húmidos, distribuidas pela fachada atlântica da Península Ibérica (RIVAS-MARTINEZ et al., 1991). Associadas à perturbação dos bosques da Quercion broteroi e Quercion robori-pyrenaicae, evidenciam boa representatividade na paisagem Carpetano-Ibérico-Leonesa (Costa et al., 2003).

\section{Comunidade basal de Cytisus striatus}

Situação ecológica, estrutura e representatividade na área em estudo

Formações arbustivas altas e densas associadas a uma dinâmica sucessional progressiva em parcelas abandonadas pela prática agrícola. Estes matagais heliófilos, de crescimento rápido, săo favorecidos pelo abandono agrícola, uma vez que a disponibilidade de solos profundos com textura equilibrada e boa drenagem fomenta o restabelecimento rápido e eficaz da leguminosa dominante.

Dominantes na paisagem resultante de abandono, são substituidas pelo giestal branco em solos pobres de substratos graníticos, que implicam uma escassez de recursos hidricos mais pronunciada no periodo estival. Em solos de génese aluvio-coluvial ou coluvial de base de vertente cede perante uma comunidade dominada por Adenocarpus complicatus s.l., que integra Ulex europaeus subsp. latebracteatus; cuja resumida representatividade está associada à manutenção do uso agrícola neste tipo de solos. É mais frequente a definição de situações em que o dominio de Cytisus striatus é acompanhado por Adenocarpus sp. (Tab.1 inv. $1,16,17,18)$.

\section{Sintaxonomia, sindinâmica e variabilidade florística}

Correspondendo a um desvio na dinâmica serial no âmbito do Holco mollis-Quercetum pyrenaicae sigmetum, por perturbação agricola, substitui as comunidades de Lavandulo sampaianae-Cytisetum multiflori nos solos abandonados com melhores potencialidades edáficas, onde se inicia uma sucessão secundária progressiva muito rápida, favorecida pelas potencialidades edáficas e pelo carácter heliófilo de Cytisus striatus.

Os giestais dominados por Cytisus striatus inventariados interpretam-se como um desvio em relação ao sintaxon subserial Ulici latebracteati-Cytisetum striati Rivas-Martinez ex J. C. Costa, Izco, Lousã, C. Aguiar e Capelo 2000, em relação ao qual apresentam um acentuado empobrecimento florístico. Além do tributo da perturbação agrícola para o empobrecimento destas comunidades altifruticetas, deve evidenciar-se a contribuição dada pela posição de transição da área em estudo, entre o luso Ulici latebracteati-Cytisetum striati e o galaico-asturiano Ulici europaei-Cytisetum striati Rivas-Martinez ex T. E. Diaz e F. Prieto 1994. O próprio carácter finícola que o primeiro sintaxa assume na área em estudo, resumido a enclaves particularmente húmidos, favorece essa situação, típica de limites corológicos (FoCAULT, 1982), uma vez que a sua ocorrência no sector setentrional do território está associada ao óptimo Galaico-Português (AGUIAR, 2001). As condições supramediterrâneas sub-húmidas a húmidas da área em estudo não favorecem a definição desta associação, embora se identifique alguma proximidade florística e estrutural nas comunidades definidas a maior altitude, onde dispōem de totais pluviométricos mais elevados.

Estes giestais são frequentemente precedidos por uma etapa arbustiva pioneira aberta e rasteira dominada por Cistus psilosepalus, a qual, nos sectores de maior altitude $(800$ - $1000 \mathrm{~m})$, pode ser acompanhada por Halimium alyssoides. Além das cistáceas, e pelo facto de ser uma comunidade aberta, estão ainda presentes espécies anuais (Ornitopus sp., Andryala integrifolia, Jasione montana) e gramíneas vivazes (Dactylis glomerata, Arrhenatherum bulbosum). Estas comunidades instalam-se decorridos 4-5 anos após o abandono agrícola da parcela, estando a sua degradação favorecida pela rápida instalação do giestal.

Dada a elevada densidade que estes giestais apresentam, apenas em condição de orla é possivel encontrar taxa associados ao Ulici latebracteati-Cytisetum striati, como Ulex europaeus subsp. latebracteatus, o que se nos afigura como um elemento fiável para relacionar as comunidades inventariadas com aquele sintaxon, entendido como diferencial em relação à associação galaico-asturiana. Em solos agrícolas de menores potencialidades verifica-se um ajuste estrutural no sentido de comunidades mais abertas, a par de um enriquecimento em espécies do Ulici-Cytisetum, como Ulex minor, reforçando a ligação entre a associação e as comunidades basais inventariadas, embora apenas um inventário apresentado na Tabela I se possa filiar claramente nesta associação (inv.1), com grande proximidade às situações descritas para o noroeste do território continental por CosTA et al., (2000). 
Tabela I

Com. basal de Cytisus striatus

(Ulici europaei-Cytision striati, Cytisetalia scopario-striati, Cytisetea scopario-striati)

\begin{tabular}{|c|c|c|c|c|c|c|c|c|c|c|c|c|c|c|c|c|c|c|}
\hline$N^{\circ}$ de ordem & 1 & 2 & 3 & 4 & 5 & 6 & 7 & 8 & 9 & 10 & 11 & 12 & 13 & 14 & 15 & 16 & 17 & 18 \\
\hline Altitude (m) & 399 & 437 & 640 & 482 & 439 & 800 & 910 & 720 & 815 & 782 & 782 & 829 & 648 & 710 & 437 & 561 & 457 & 364 \\
\hline Cobertura (\%) & 100 & 85 & 100 & 98 & 95 & 90 & 100 & 100 & 95 & 85 & 90 & 100 & 90 & 90 & 95 & 100 & 100 & 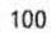 \\
\hline Área $\left(m^{2}\right)$ & 100 & 100 & 60 & 100 & 100 & 100 & 80 & 80 & 100 & 100 & 100 & 100 & 100 & 100 & 100 & 100 & 100 & \\
\hline Rocha & clv & $x / g r$ & $x$ & $x$ & $x / g r$ & $g r$ & $x$ & gr & $x$ & gr & $\mathrm{gr}$ & $x$ & $x$ & $x$ & $\mathrm{gr}$ & $g r$ & $x$ & \\
\hline Exposição & - & - & W & NE & - & - & - & - & W & - & $\cdot$ & - & $E$ & $\mathrm{~S}$ & - & $\cdot$ & $\cdot$ & \\
\hline Declive $(\%)$ & - & - & - & - & - & - & - & - & 5 & - & - & - & 7 & 7 & - & - & $\cdot$ & \\
\hline
\end{tabular}

Características de associação e sintaxa superiores

Adenocarpus complicatus

Cytisus striatus

Ulex latebracteatus

Orobanche rapum-genistae

Genista florida

Ulex minor

Companheiras

Agrostis capilaris

Quercus pyrenaica

Senecio sylvaticus

Rubus sp.

Erica arborea

Cistus psilosepalus

Holcus mollis

Avenula sulcata

Dactylis glomerata

Digitalis purpurea

Senecio minor

Frangula alnus

Pteridium aquilinum

Pinus pinaster

Agrostis castellana

Halimium alyssoides

Costanea sativa

Pterospartum lasianthum

Prunus avium

Antoxanthum odoratum

Avenula sulcato

Hypericum linarifolium

Rumex angiocarpus

Micropyrum tenellum

Teesdalia nudicaulis

Conopodium marizianum

Jasione montana

Briza maxima

Echium lusitanicum

Erica australis

Outras companheiras: inv. 1 Vicia sativa +, Umbilicus rupestris +; inv. 4 Arbutus unedo 1, Lithodora prostrata +; inv. 5 Cytisus scoparius 1 , Erica umbellata +; inv 10 Geranium robertianum +; inv. 11 Anarrhinum duriminum +, Agrostis truncatula +; inv. 12 Pseudarrenatherum longifolium +, Agrostis curtisii +; inv. 13 Daphne gnidium +, Sanguisorba minor +; inv. 14 Cistus salvifolius 1, Calluna vulgaris +, Lavandula sampaioana +, Lonicera hispânica +; inv. 17 Rubia peregrina; inv. 18 Vulpia bromoides +, Bromus tectorum +, Lactuca sp. +.

Localidades: 1. Chaves: Vilela Seca, veiga; 2 - Chaves: Vilela Seca, cruzamento para Vilarelho; 3 - Chaves: S. Juliâo, estanheira velha; 4 - Chaves: S. Pedro de Agostém, vinha; 5 - Chaves: Vilela Seca, cruzamento para Vilarelho; 6 - Chaves: France, por cima da estrada nacional; 7 - Chaves: Brunheiro; 8 - Chaves: cruzamento Castelōes/S.Caetano; 9 Chaves: Mosteiro de Cima, cruzeiro; 10 - Chaves: Castelo de Monforte; 11 - Chaves: Vila Nova, saida; 12 - Chaves: Vila Nova, vèrtice geodésico; 13 e 14 - Chaves: S. Lourenço, Lameiro Novo; 15 - Chaves: Pastoria, entrada; 16 - Chaves: Sanjurge, rest. Mentiras; 17 - Chaves: Bóbeda; 18 - Chaves: Vila Meã - ao pé do Tãmega.

Litologia: gr - granito, x - xisto, clv - coluviảo. 
O decorrer da sucessão implica a degradação destes giestais, favorecendo o reforço em poáceas (Agrostis sp.) e a entrada de taxa com um carácter mais heliófilo, posteriormente expulsas pela implantaçāo progressiva do carvalhal. Em solos mais profundos, normalmente associados a condiçōes depressionárias e substratos xistentos, pode verificar-se um reforço importante em Agrostis capilaris (inv. 16), enquanto que as comunidades instaladas nos sectores de maior altitude do flanco oriental da bacia manifestam um reforço em Holcus mollis, o que parece estar associado à presença de condiçōes bioclimáticas húmidas, favoráveis à implantação da formação potencial arbórea de Holco mollis-Quercetum pyrenaicae.

Nos sectores de maior altitude, associados à superficie fundamental da meseta, o aumento dos totais pluviométricos favorece a definição de um ombroclima húmido, permitindo a entrada nestes giestais do taxon Genista florida subsp. polygaliphylla (inv. 7 e 12), que ao remeter para uma ligação floristica ao Cytiso striati-Genistetum polygaliphyllae, interpretado como subserial dos carvalhais de Holco mollis-Quercetum pyrenaicae, reforça a ideia da presença potencial destes bosques. Verificável em ambos os flancos da depressão, esta situação tem continuidade pelos sectores supramediterrâneos da Serra da Padrela', a altitudes superiores aos $800 \mathrm{~m}$, onde $\mathrm{o}$ abandono agricola pode mesmo favorecer a instalação de uma comunidade dominada por Genista polygaliphylla, mantendo grande parte dos atributos identificados para as comunidades da área em estudo dominadas por Cytisus striatus, nomeadamente a pobreza florística e a estrutura.

Lavandulo sampaioanae-Cytisetum multiflori $\mathrm{Br}$.-Bl., P. Silva e Rozeira 1964

Situação ecológica, estrutura e representatividade na área em estudo

As comunidades inventariadas são interpretadas como uma variante floristicamente empobrecida do Lavandulo sampaioanae-Cytisetum multiflori Br.-Bl., P. Silva e Rozeira 1964, já por si uma associação caracterizada por acentuada pobreza florística (RIVAS- MARTINEZ, 1981). Esta associação representa um giestal heliófilo dominado por Cytisus multiflorus (L’Her.) Sweet, de óptimo carpetano-ibéricoleonês e beiraduriense meso-supramediterrâneo para

1. Comunidades inventariadas na proximidade de Matosinhos (Carrazedo de Montenegro) e Zimảo (V. P. Aguiar). ombroclimas sub-húmidos a húmidos (COSTA, 2003), cuja corologia reflete um reforço da influência da continentalidade em relação à associação de aptências atlânticas Ulici lactebracteati-Cytisetum striati.

$\mathrm{Na}$ área em estudo, estas comunidades apresentam alguma variabilidade, resultado de diferentes tipos de perturbação e condições ecológicas. Como fitocenose primocolonizadora de solos abandonados pela agricultura, ocorre tambèm em biótopos perturbados por fogo e aproveitamento de mato. Assegura maior representatividade nas vertentes mesomediterrâneas sub-húmidas, onde as condiçōes meso-xerófilas, fomentadas pelos declives acentuados e solos pobres, săo reforçadas pela disseminação de afloramentos graniticos, o que thes assegura elevada resiliência. Nestas situaçōes, ecologicamente mais exigentes, apresentam menor cobertura e maior riqueza florística, evidenciando um reforço em taxa da Quercetea ilicis e da Cisto-Lavanduletea (RODRIGUES, 2005). Nesta análise, a atenção é apenas dispensada à ocorrência relacionada com o abandono agricola, em situações em que a utilização do solo esteve associada, principalmente, ao cultivo de centeio. Por serem parcelas com solos mais profundos, estas comunidades evidenciam maior densidade e pobreza floristica, em relação às instaladas em biótopos mesoxerófilos.

\section{Sintaxonomia e variabilidade floristica}

Interpretadas no âmbito do Lavandulo sampaioanae-Cytisetum multiflori, apresentam um dominio absoluto de Cytisus multiflorus (L'Her.) Sweet, com representatividade resumida de Lavandula stoechas subsp. sampaioana Rozeira (inv.2). Estas comunidades representam uma variante floristicamente empobrecida de solos abandonados pela utilização agrícola, quando comparadas com giestais brancos instalados em biótopos não perturbados por este tipo de utilização.

Em solos mais delgados, onde o giestal de Cytisus multiflorus apresenta menor cobertura, verifica-se um reforço de Lavandula sampaioana, situação que é pouco frequente em parcelas abandonadas pela prática agrícola, uma vez que o seu comportamento heliófilo não é compatível com a elevada densidade dos giestais brancos que aí se definem. Por serem comunidades mais abertas permitem ainda a entrada de espécies de arrelvado (Agrostis castellana, Avenula sulcata).

A ausência de Genista hystrix na área em estudo remete para a ausência das associaçōes Carpetano-Ibérico-Leonesas com afinidades ecológicas, como é a 
Genisto hystricis-Cytisetum multiflori e a LavanduloGenistetum hystricis, associadas por ORTIz et al. (1991) no vale do Sil a uma dinâmica regressiva no domínio de bosques perenifólios de Genisto hystricisQuercetum rotundifoliae.

\section{Contactos catenais, sinfitossociologia e sindi-nâmica}

Desenvolve-se em solos arenosos, mais pobres em matéria orgânica que os que permitem o desenvolvimento das comunidades de Cytisus striatus, normalmente cambissolos dístricos resultantes da alteração dos granitos (NAVARRo et al., 1987). No andar supramediterrâneo e horizonte superior mesomediterrâneo estas comunidades podem incluir no seu fundo florístico elementos da Calluno-Ulicetea, dado o contacto que se verifica com comunidades da Ericenion umbellatae, nomeadamente pela presença de Pterospartum lasianthum (inv. 5). Após abandono pela actividade agricola verifica-se a instalação de uma comunidade arbustiva pioneira muito aberta, onde a fraca cobertura determinada pela presença esparsa de Lavandula sampaioana, por vezes acompanhada por Cistus psilosepalus, permite a manutenção de um arrelvado anual. Esta cistácea apresenta, no entanto, reduzida importância, comparativamente à mesma situação sucessional em solos mais espessos, associados à ocorrência potencial de comunidades dominadas por Cytisus striatus.

As comunidades inventariadas, associadas a uma dinâmica secundária progressiva, representam um desvio na série encabeçada por bosques de HolcoQuercetum pyrenaicae. No sector mesomediterrâneo superior associam-se a uma versão mais termófila e sub-húmida destes carvalhais, mais próxima ao conceito do Genisto falcatae-Quercetum pyrenaicae, ou interpretada como uma variante mais seca da primeira associação, uma vez que podem ser entendidas como sinónimas em termos sintaxonómicos, dado o elevado número de taxa característicos partilhados (LADERO et. al., 2003; PINTOGOMEs et. al., 2003; AGUIAR, 2001). Em sectores não associados a perturbação agrícola, biótopos mesoxerófilos, o giestal branco está associado a bosques potenciais mistos, que traduzem a interpenetração de bosques perenifólios mesomediterrâneos de Quercus Suber (Physospermo cornubiensis-Quercetum suberis Rivas-Martinez 1987) e os bosques climatófilos supramediterrâneos de Quercus pyrenaica Willd. (Holco mollis-Quercetum pyrenaicae).
Tabela II

Com. basal de Cytisus multiflorus

(Ulici europaei-Cytision striati, Cytisetalia scopario-striati, Cytisetea scopario-striati)

\begin{tabular}{lccccc}
\hline$N^{\circ}$ de ordem & 1 & 2 & 3 & 4 & 5 \\
Altitude $(\mathrm{m})$ & 783 & 785 & 800 & 856 & 575 \\
Cobertura $(\%)$ & 95 & 75 & 100 & 100 & 100 \\
Área $\left(\mathrm{m}^{2}\right)$ & 100 & 75 & 100 & 100 & 70 \\
Rocha & gr & gr & gr & gr & gr \\
Exposiçāo & $\mathrm{S}$ & W & - & - & - \\
Inclinação $(\%)$ & 5 & 5 & - & - & .
\end{tabular}

Características de associação e sintaxa superiores

Cytisus multiflorus

Lavandula sampaioana

Orobanche rapum-genistae

Cytisus striatus

Ulex minor

Erica arborea

Companheiras

Agrostis castellana

Quercus pyrenaica

Halimium alyssoides

Pterospartum lasianthum

Pteridium aquilinum

Anthoxanthum aristatum

Avenula sulcata

Halimium viscosum

Senecio minor

Senecio sylvaticus

Outras companheiras: inv. 1: Anarrhinum belidifolium +; inv. 2: Jasione montana + , Sanguisorba minor + , Tuberaria guttata + , Geranium molle +, Vulpia bromoides +, Bromus driandrus +, Bromus ordeaceus +, Galium sp. +, Veroina agrestis +, Bellis perennis + Cardamine hirsuta +, Cerastium glormetatum +, Hispidella hispanica +, Teesdalia nudicaulis +; inv. 4: Umbilicus rupestris +, Peribalia involucrata + , Rumex angiocarpus +; inv. 5: Erica umbellata + , Polygala microphyla + .

Localidades: 1, 2 e 3 - Chaves: France, Estrada Nacional; 4 - Chaves: Castelo de Monforte; 5 - Chaves: Sanjurge - Rest. Mentiras.

Litologia: gr: granito.

\section{Conclusões}

0 abandono agrícola nesta área do país, tal como se pode comprovar praticamente em todo o interior Norte e Centro de Portugal Continental, favorece a instalação de comunidades arbustivas dominadas por espécies do género Cytisus, podendo as mesmas ser acompanhadas por outros taxa, em função das condições ecológicas presentes. A paisagem agrícola sujeita a abandono massivo é dominada por giestais de Cytisus striatus, desenvolvidos em solos mais profundos e produtivos, quer seja em substratos 
graníticos ou xistentos. Já as comunidades dominadas por Cytisus multiflorus ocupam exclusivamente solos graníticos, cambissolos dístricos pouco profundos e de textura arenosa, que implicam um défice hídrico mais acentuado no período estival. Sendo comunidades basais, que evidenciam um evidente empobrecimento florístico, são interpretadas como desvios na sucessão em relação às comunidades subseriais do Holco mollisQuercetum pyrenaicae sigmetum, em biótopos perturbados pela prática agricola e posteriormente abandonados.

Tendo em conta a representatividade que estas comunidades assumem, e a dinâmica que imprimem à paisagem, frequentemente perturbada pelo fogo, é imperiosa a definição de medidas direccionadas para a sua gestão. Apesar de marginais para o aproveitamento agricola, estas áreas, massivamente afectadas pelo abandono, apresentam condições adequadas ao aproveitamento florestal. A este respeito, parece viável apostar na recuperação dos bosques autóctones, tendo em conta a satisfatória taxa de regeneração do carvalho negral (Quercus pyrenaica Willd.).

\section{Referências Bibliográficas}

Aguiar, C. (2001) - Flora e Vegetação da Serra da Nogueira e do Parque Natural de Montesinho. Tese de Doutoramento, Instituto Superior de Agronomia, Lisboa.

CASTROVIEJo, S. (coord.) (1986-2000) - Flora Iberica. Vols. I-VIII. Real Jardín Botánico de Madrid, CSIC. Madrid.

CIPC (CALIFORNIA INVASIVE PLANT COUNCIL) (2006) - California Invasive Plant Inventory [Online]. Cal-IPC. Disponivel:http://www.calipc.org/ip/inventory/pdf/ln ventory2006.pdf [2 Março 2007].

Correia, T. P.; Breman, B.; Jorge, V. e Dneboská, M. (2006) Estudo sobre o abandono em Portugal. Análise das dinâmicas da ocupação do solo, do sector agrícola e da comunidade rural [Online]. Universidade de Évora. Disponivel:

http://www.min-agricultura.pt/temas/agricultura/Estudo Abandono.pdf [15 Novembro 2006].

Costa, J. C.; Aguiar, C.; Capelo, J.; Lousä, M. e Neto, C. (1998) "Biogeografia de Portugal Continental". Quercetea, vol. 0, pp. 5-56.

Costa, J.; Capelo, J. H.; lousä, M; Antunes, J. H. C.; Aguiar, C.; IzCO, J. e LADERO, M. (2000) - "XXVIII: Nota acerca dos giestais da aliança Ulici europaei-Cytision striati RivasMartinez, Báscones, T. E. Díaz, Fernández-González \& Loidi em Portugal continental". Silva Lusitana, vol. $8 /$ n..$^{\circ}$, pp. $120-128$.
Costa, J.; Aguiar, A.; CApelo, J.; Lousã, M.; Antunes, J. H. S. C.; Honrado, J. J.; Sevillano, J. I. e Alvarez, M. L. (2003) - "A classe Cytisetea scopario-striati em Portugal Continental". Quercetea, vol.4, pp. 45-70.

FOCAULT, B. (1982) - "Appauvrissement des syntaxons aux limites chorologiques". Lazaroa, vol. 3, pp. 75-100.

Franco, J. A. e Rocha Afonso, M. L. (1998) - Nova Flora de Portugal (Continente e Açores) - GRAMINEAE . vol III, fasc. II. Escolar Editora. Lisboa.

GèHu, J. M. e Rivas-Martinez, S. (1981) - "Notions fondamentales de phytosociologie". In: DIERSCHKE, H. (ed.) Syntaxonomie, Ber Int. Symp. Int. Vereinigung Vegetatiousk. J. Cramer, Vaduz, pp. 5 - 33.

KOPECKÝ, K. e S. HEJNÝ (1974) - "A new approach to the classification of anthropogenic plant communities". Vegetatio, vol. 29, pp. 17-21.

Ladero, M.; Amor, A.; Santos, M. T.; Sánchez, M. E. e Ferro, G. (2003) - "Robledales supramediterráneos de ombroclima subhúmedo en los sectores Salmantino y Lusitano-Duriense". Quercetea, vol. 4, pp. 5-11.

Llorente Pinto, J. M. e Luengo Ungidos, M. A. (1986) - "El abandono de las tierras: significado y gestión de las etapas de sucesión secundaria. El ejemplo de los relieves paleozoicos del Oeste de Castilla y León". Monografies de l'EQUIP, vol. 2, pp. 105-114.

marcos, E.; Tárrega, R. e luis-Calabuig, E. (2004) - "Interactions between mediterranean shrub species eight years after expermimental fire". Plant Ecology, vol. 170, pp. 235 . 241.

Navarro, F.; Sánchez-Anta, M. A.; González-Zapatero, M. A.; Gallego, F.; Elena, J. A.; Valle, C. J. e lópez-Blanco, L. (1987) - "Piornales y retamales meso y supramediterráneos salmantinos y zamoranos". Lazaroa, vol. 7, pp. 337-349.

Pérez-Chácon, E. e VABRe, J. (1988) - "Abandono agropastoril y recolonización vegetal: el papel de las leñosas como indicadoras del estadio de regeneración vegetal y de la edad del abandono (Haut Couserans-Pirineo Central Frances)". Cuadernos I. Geografica, vol. 14/n. ${ }^{\circ} 1-2$, pp. 99-120.

Pinto-Gomes, C. J.; Alvarez, M. L.; Gonçalves, P. C. C. e PaivaFerreira, R. (2003) - "Contribuiçāo para o estudo dos bosques supramediterrânicos de Quercus pyrenaica na Reserva Natural da Serra da Malcata". Quercetea, vol. 4, pp. 13-21.

Rivas-Martínez, S. (1981) - "Sobre la vegetación de la Serra da Estrela (Portugal)". An. Real Acad. Farmacia, vol. 47, n. ${ }^{\circ}$, pp. $435-480$.

Rivas-martinez, S.; Báscones, J. C.; Diaz, T. E.; FernándezGonzalez, F. e LoIdI, J. (1991) - Vegetación del Pirineo occidental y Navarra. Itinera Geobot., vol. 5, pp.5-455. 
Rivas-Martínez, S.; Fernandez-González, F. e Loldi, J. (1999) "Checklist of plant communities of Iberian Peninsula, Balearic and Canary Islands to suballiance level". Itinera Geobotanica, vol. 13, pp. 353-451.

Rivas-Martinez, S.; Fernández-González, F.; Loldi, J.; Lousā, M. e PEN/S, A. (2001) - "Syntaxonomical checklist of vascular plant communities of Spain and Portugal to association level". Itinera Geobot., vol. 14, pp. 5-341.

Rivas-Martinez, S.; Dlaz, T. E.; Fernández-González, F.; izco, J.; LoIDI, J.; LousĀ, M. e PENAS, A. (2002) - "Vascular plant communities of Spain and Portugal. Addenda to the syntaxonomical checklist of 2001". Itinera Geobotanica, vol. 15,1 .992.
Rodrigues, A. (2005) - Vegetaçâo Actual e Potencial num Contexto de Elevada Perturbaçāo: a Depressão de Chaves. Tese de Mestrado, Faculdade de Letras da Universidade de Coimbra, Coimbra, 198 p.

Tarrega, R.; Calvo, L. e Trabaud, L. (1992) - "Effect of high temperatures on seed germination of two woody Leguminosae". Vegetatio, vol. 102, pp. 139-147. 